\title{
Microscopy Observation of Lithium Deposition Behavior on Graphene Matrix
}

\author{
Tara Foroozan $^{1}$, Soroosh Sharifi-Asl ${ }^{1}$ and Reza Shahbazian-Yassar ${ }^{1}$ \\ 1. Mechanical and industrial Engineering Department, University of Illinois at Chicago, Chicago, IL.
}

Lithium metal (Li) with ultrahigh specific capacity $\left(3860 \mathrm{mAh} \mathrm{g}^{-1}\right)$ is the most wanted anode material for realization of light and high-power batteries. However, dendritic deposition of Li and the corresponding safety hazards has prevented the commercialization of Li-metal batteries (LMB). Li dendrites initiate mainly on anode surface protuberances with enhanced electric field. In addition, low mechanically stable solid electrolyte interphase (SEI) gives rise to the unwanted dendritic growth of Li and its poor performance [1]. Therefore, improving the uniformity of Li-ions flux and mechanical stability of SEI are critical approaches towards regulation of Li-metal anode to be used in next generation of batteries.

During the past decade, researchers have taken different approaches in utilization of various materials properties to prevent the dendritic plating of Li. Graphene, the wonder material, is among the most promising materials used for impeding the dendritic deposition of Li and improving the electrochemical performance of Li metal batteries [2-4]. However, the detailed understanding of the mechanism behind this outperformance has not been studied so far. Yan et al. [2] and Xie et al. [3] have suggested that, chemical vapor deposition (CVD) graphene can combat the dendritic deposition of Li. They have hypothesized that ultrathin layers of Gr can act as artificial SEI, which can permit the diffusion of Li-ions to the $\mathrm{Cu}$ substrate, where the Li deposition occurs. However, there is no detailed discussion on why Liions should pass the Gr sheets and deposit on the electrode surface and not on the highly electrically conductive Gr and its active edges. Alternatively, Zhang et al. [4] have proposed that drum like Gr with ultrahigh surface area and electrical conductivity can be a desirable matrix, which reduces the effective electrode current density, for uniform Li deposition.

It is expected that the Li nucleation and growth mechanism change by changing the deposition matrix. However, no detailed study has been performed to evaluate nucleation and growth of Li dendrites in the presence of 2D materials coating. Considering that microscopy techniques are widely used to evaluate the Li deposition morphology, herein we have used optical and scanning electron microscopy techniques to evaluate the Li nucleating and growth behavior in case of Gr-coated electrodes to better understand the effect $\mathrm{Gr}$ on the Li nucleation and growth mechanism. The as grown CVD graphene on $\mathrm{Cu}$ samples were directly used to fabricate $\mathrm{Li} / \mathrm{Cu}$ transparent electrochemical cells (Figure 1A). Interestingly, unlike typical dendritic deposition of $\mathrm{Li}$ on bare $\mathrm{Cu}$, a uniform and non-localized $\mathrm{Li}$ deposition was observed on the graphene-modified $\mathrm{Cu}$ electrodes (Figure 1B). As it can be seen in the inset of Figure 1B, donut shaped Li dendrites were grown in small areas $(<5 \%)$, which has a distinct morphology from that of grown on an un-modified electrode. Moreover, SEM observations demonstrates more details of modified Li-deposition in presence of graphene layer. As can be seen from Figure 1C, dense and smooth deposition of Li in form of continuous colonies, covering the whole surface, has altered the typical dendritic growth of lithium. In addition, higher magnification SEM images (Figure 1D) show that deposited Li colonies are consisting of dense, spherical shape Li grains with uniform size of $\sim 800 \mathrm{~nm}$ in diameter (inset of Figure 1D) that form a final non-dendritic Li film. This covering bump like Li deposition evidences the uniformly distributed current density on the graphene layer. The observed spherical Li nucleation behavior is very different from what we observe in case of bare $\mathrm{Cu}$ electrode as evidenced by Figure 1E. As it can be seen in the Figure $1 \mathrm{E}$ inset, non-uniform and dendritic Li deposition will happen from early stages of Li deposition in case of bare electrodes, which eventually grows into large dendrites that can penetrate through the separator 
and short circuit the battery. In a recent work, has proposed the use of cryo-TEM for studying the battery sensitive components like Li dendrites. Exploiting this capability, we are further investigating the underlying mechanism for the observed uniform $\mathrm{Li}$ nucleation and growth utilizing cryo-TEM microscopy. Studying the associated SEI composition as well as the preferred Li growth directions can help us understand how 2D materials affect the morphology of electrodeposited $\mathrm{Li}$ and figure out if the $\mathrm{Li}$ plating occurs underneath or on top of the highly conductive Gr film.

\section{References:}

[1] Lang, J., Qi, L., Luo, Y., Energy Storage Mater. 7 (2017), p. 115.

[2] Lu, Z., Zhou, Y., Liang, Z., Nano Lett. 14 (2014), p. 6016.

[3] Xie, K., Wei, W., Yuan, K., ACS Appl. Mater. Interfaces 8, (2016), p. 26091.

[4] Zhang, R., Cheng, X. B., Zhao, C. Z., Adv. Mater. 28, (2016), p. 2155.
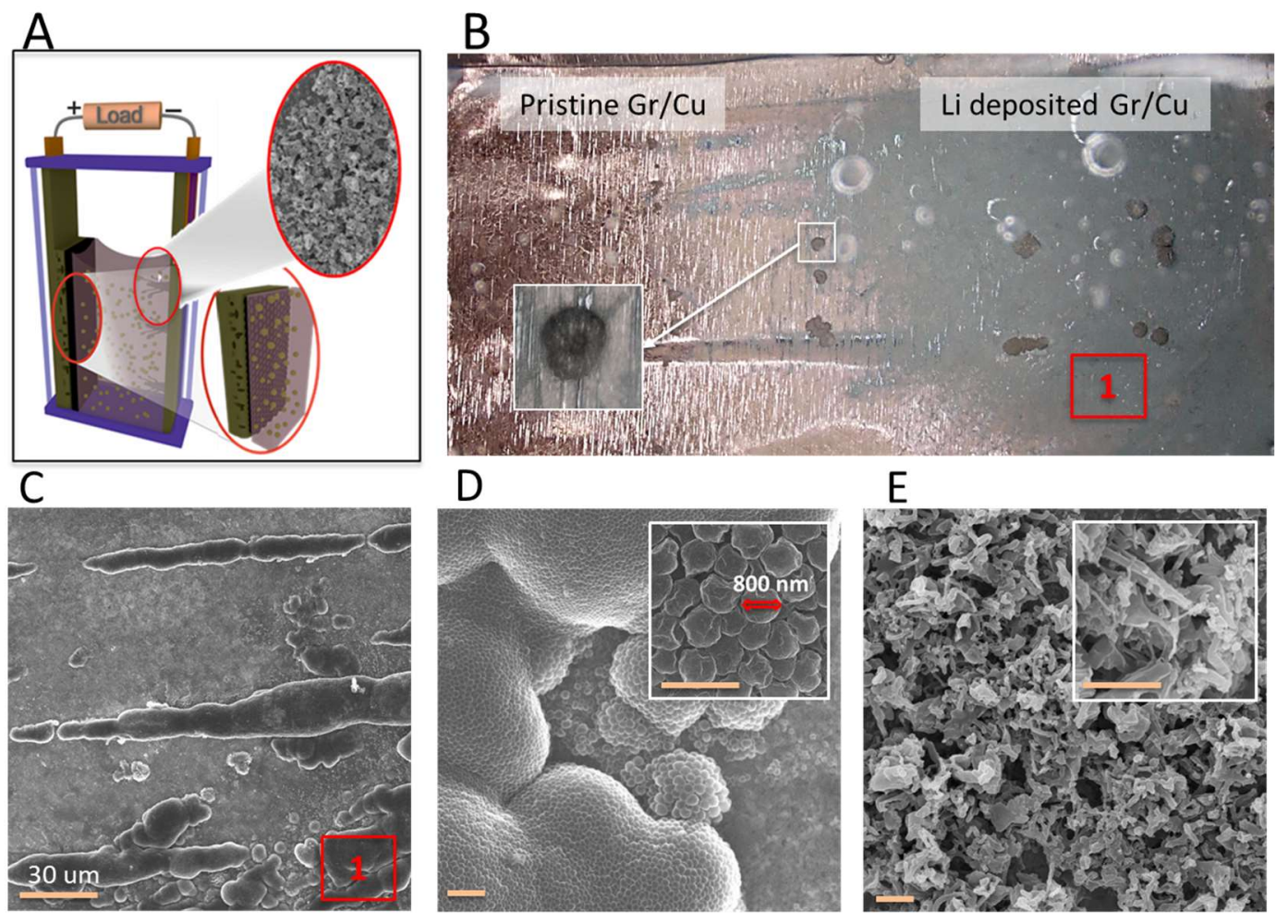

Figure 1. Li deposition comparison in case of bare and Gr- modified Cu electrodes. (A) Schematic of the quartz battery cell used for studying the Li deposition mechanism in case of $\mathrm{Gr}$ modified $\mathrm{Cu}$ electrode. Magnified insets show the Li deposition in case of Gr modified and bare electrodes. (B) Optical image obtained from the $\mathrm{Gr} / \mathrm{Cu}$ electrode after $2 \mathrm{mAh}$ of Li deposition in the quartz halfcell battery. (C) Low magnification SEM image obtained from the area (1) in Figure B shows the morphology of Li deposition in case of $\mathrm{Gr} / \mathrm{Cu}$ electrode. (D) Higher magnification SEM image shows the spherical Li deposition covering the whole electrode surface. Inset shows the uniform $\mathrm{Li}$ spheres with size of $\sim 800 \mathrm{~nm}$. (E) SEM image obtained from control Cu electrode shows typical dendritic deposition of Li. Inset is the higher magnification image of non-uniform Li dendrites. Scale bars in images D and $\mathrm{E}$ are $2 \mu \mathrm{m}$. 